\title{
Clinical trials update: recent and ongoing studies in anticoagulation for atrial fibrillation
}

This article was published in the following Dove Press journal:

Open Access Journal of Clinical Trials

26 August 2013

Number of times this article has been viewed

\section{James W Wisler}

Division of Cardiology, Duke University Hospital, Durham, NC, USA
Correspondence: James W Wisler

Division of Cardiology, 7400 Duke University Hospital, 230I Erwin Road,

Durham, NC 277I0, USA

$\mathrm{Tel}+19196844703$

Fax + I 9196848875

Email jim.wisler@duke.edu

\begin{abstract}
Atrial fibrillation is the most common sustained cardiac arrhythmia in the adult population, with a marked increased risk associated with age. Perhaps the most devastating complications of atrial fibrillation include acute ischemic stroke or systemic embolization. Vitamin $\mathrm{K}$ antagonists such as warfarin have served as the primary pharmacologic agent for the prevention of these thrombotic complications. Despite the widespread use of vitamin $\mathrm{K}$ antagonists, their effectiveness is hindered by several factors, including delayed onset of action, multiple food and drug interactions, onerous monitoring and dosing regimens, and a narrow therapeutic window. To address these limitations, multiple novel oral anticoagulants targeting thrombin or factor Xa have progressed through clinical development or received regulatory approval for clinical use in recent years. These agents offer the potential for enhanced efficacy, as well as an improved safety profile. Here, the phase II and III clinical trial data detailing the potential risks and benefits of these agents are reviewed.
\end{abstract}

Keywords: atrial fibrillation, factor Xa inhibitor, direct thrombin inhibitor, clinical trial

\section{Introduction}

Atrial fibrillation (AF) is the most common sustained cardiac arrhythmia worldwide, with a lifetime risk of nearly $25 \%$ in adults. ${ }^{1}$ The risk of stroke is markedly elevated from $1 \%$ to $20 \%$ annually among adults with nonvalvular $\mathrm{AF}^{2} \mathrm{AF}$ is also associated with increased in-hospital mortality in both atherothrombotic and cardioembolic stroke patients. ${ }^{3}$ Vitamin $\mathrm{K}$ antagonists (VKAs) have been the primary pharmacologic therapy for the prevention of thrombotic complications in patients with AF for decades. ${ }^{4}$ Treatment with a VKA has a class I recommendation for all patients with nonvalvular AF who are at elevated risk of stroke or systemic embolization. ${ }^{4}$ These recommendations are supported by an observed reduction of up to $62 \%$ in the risk of stroke in patients with nonvalvular AF treated with VKAs. ${ }^{5}$

Despite their effectiveness in the prevention of thrombotic complications in patients with AF, VKAs are underutilized, and in clinical practice they may be relatively ineffective in these patients. ${ }^{6,7}$ This observation has been attributed to several factors intrinsic to VKAs, including a delayed onset of anticoagulant effect, narrow therapeutic window, early prothrombotic effect observed with therapy initiation, burdensome drug monitoring including frequent laboratory testing, and unpredictable anticoagulant effects secondary to numerous medication and food interactions and genetic polymorphisms. ${ }^{8}$

To address the limitations and complexities associated with VKAs, in recent years multiple novel oral anticoagulants, including those targeting thrombin or factor 
Xa directly, have progressed through clinical development or received regulatory approval in this patient population and entered into clinical practice. In particular, dabigatran (Pradaxa; Boehringer Ingelheim Pharmaceuticals, Ingelheim, Germany), apixaban (Eliquis; Bristol-Myers Squibb, New York, NY, USA), and rivaroxaban (Xarelto; Johnson and Johnson, New Brunswick, NJ, USA, and Bayer Schering Pharma, Berlin, Germany) were recently approved by the US Food and Drug Administration (FDA) for the prevention of stroke and systemic embolism in patients with nonvalvular AF.

These agents have been evaluated in three major clinical trials comparing warfarin to the respective novel anticoagulant in patients with nonvalvular $\mathrm{AF}^{9-11}$ (Table 1), as well as one comparing acetylsalicylic acid to apixaban in patients for whom warfarin was deemed unsuitable. ${ }^{12}$ In addition to these agents, two other factor Xa inhibitors - betrixaban (Portola Pharmaceuticals, San Francisco, CA, USA) and edoxaban (Lixiana; Daiichi Sankyo, Tokyo, Japan) have reached late stages of clinical development and been investigated in phase II randomized, controlled trials. ${ }^{13,14}$ Here, the key aspects of the pivotal trials for each of these agents are reviewed.

\section{Direct thrombin inhibitors Dabigatran}

Dabigatran etexilate is an oral, direct thrombin inhibitor recently approved for use in patients with nonvalvular AF. This medication is supplied as a prodrug, and after ingestion is quickly converted to its active metabolite. ${ }^{15}$ Peak plasma concentrations are observed in approximately 2-3 hours, and this agent has a half-life of 12-14 hours (Table 2). Nearly $80 \%$ of ingested dabigatran is eliminated via the renal system, while the other $20 \%$ is conjugated and excreted via the biliary system. Dabigatran does not interact with hepatic cytochrome P450 (CYP) enzymes. It does, however, interact with the efflux transporter P-glycoprotein (P-gp), ${ }^{16}$ and is therefore subject to drug interactions with agents that activate (eg, rifampin) or inhibit (eg, amiodarone, ketoconazole, quinidine, or verapamil) this transporter.

The use of dabigatran in patients with nonvalvular AF was investigated in the phase III RE-LY (Randomized Evaluation of Long-Term Anticoagulant Therapy) trial. ${ }^{11}$ In RE-LY, 18,113 patients were randomized in a prospective, randomized, open-label, blinded end-point (PROBE) design to either $110 \mathrm{mg}$ or $150 \mathrm{mg}$ of dabigatran twice daily or openlabel warfarin (target international normalized ratio [INR] 2.0-3.0). The median follow-up was 2 years. At the end of this follow-up, no significant difference was observed in the primary efficacy end point (composite of stroke or systemic embolism) between those treated with warfarin compared to those treated with $110 \mathrm{mg}$ dabigatran $(1.69 \%$ versus [vs] $1.53 \%$ per year, $P<0.001$ for noninferiority). However, the rate of the primary efficacy end point was significantly lower in the $150 \mathrm{mg}$ group compared to the warfarin group (1.11\% vs $1.69 \%$ per year, $P<0.001$ for noninferiority and superiority). The rate of major bleeding was lower with lower-dose dabigatran compared to warfarin $(2.71 \%$ vs $3.36 \%$ per year, $P=0.003$ ), and similar between the $150 \mathrm{mg}$ dose group and warfarin-treated patients $(3.11 \%$ vs $3.36 \%$ per year, $P=0.31$ ). The risk of life-threatening bleeding was significantly lower with both doses of dabigatran compared to warfarin $(1.22 \%$ and $1.45 \%$ vs $1.80 \%, P<0.05$ for all comparisons of dabigatran to warfarin). The rate of intracranial hemorrhage was also significantly reduced for each dabigatran dose group relative to warfarin-treated patients $(0.23 \%$ and $0.30 \%$ for the $110 \mathrm{mg}$ and $150 \mathrm{mg}$ dose groups,

Table I Phase III trials of novel anticoagulants in nonvalvular atrial fibrillation

\begin{tabular}{|c|c|c|c|c|}
\hline & RE-LY & ROCKET AF & ARISTOTLE & EngageAFTIMI48 \\
\hline Medication & Dabigatran & Rivaroxaban & Apixaban & Edoxaban \\
\hline Dose (mg) & 150 and I 10 mg twice daily & 20 or 15 mg* daily & 5 or 2.5 mg** twice daily & 30 and 60 mg daily \\
\hline$n$ & 18,113 & 14,264 & $|8,20|$ & $>21,000$ \\
\hline Design & PROBE & Double-blind & Double-blind & Double-blind \\
\hline \multirow[t]{2}{*}{ AF criteria } & $\mathrm{AF} \times \mathrm{I}$ & $\mathrm{AF} \times 2$ & $\mathrm{AF}$ or $\mathrm{AF} \times 2$ & $\mathrm{AF} \times \mathrm{I}$ \\
\hline & $<6$ months & $\geq \mathrm{I}$ in $<30$ days & $<12$ months & $<12$ months \\
\hline \% VKA-naive & 50 & 38 & 43 & 40 (goal) \\
\hline $\mathrm{CHADS}_{2}$ (median) & 2.2 (mean) & 3.5 & 2.1 & NA \\
\hline $\mathrm{CHADS}_{2} 3-6(\%)$ & 32.5 & 87 & 30.2 & NA \\
\hline
\end{tabular}

Notes: *Dose reduced to $15 \mathrm{mg}$ in patients with creatinine clearance of $30-49 \mathrm{~mL} /$ minute; **dose reduced to $2.5 \mathrm{mg}$ in patients with reduced drug clearance. Abbreviations: RE-LY, Randomized Evaluation of Long-Term Anticoagulant Therapy; ROCKET AF, Efficacy and Safety Study of Rivaroxaban with Warfarin for the Prevention of Stroke and Non-central Nervous System Systemic Embolism in Patients with Non-valvular Atrial Fibrillation; ARISTOTLE, Apixaban for the Prevention of Stroke in Subjects with Atrial Fibrillation; EngageAFTIMI48, Global Study to Assess the Safety and Effectiveness of Edoxaban (DU-I76b) vs Standard Practice of Dosing with Warfarin in Patients with Atrial Fibrillation; PROBE, prospective, randomized, open-label, blinded end-point evaluation; AF, atrial fibrillation; VKA, vitamin K antagonist; NA, not available. 
Table 2 Characteristics of novel anticoagulants

\begin{tabular}{llllll}
\hline Drug name & Dabigatran & Apixaban & Rivaroxaban & Betrixaban & Edoxaban \\
\hline Target & Factor Ila (thrombin) & Factor Xa & Factor Xa & Factor Xa & Factor Xa \\
Dosing & Twice daily & Twice daily & Daily & Daily & Once or twice daily \\
Half-life (hours) & $12-14$ & $8-13$ & $5-13$ & 19 & $6-11$ \\
$\begin{array}{l}\text { Renal excretion (\%) } \\
\text { Drug interactions }\end{array}$ & 80 & 25 & 66 & 17 & 35 \\
& Potent inhibitors/ & Potent inhibitors & Potent inhibitors of & Low potential & Potent inhibitors of \\
& inducers of P-gP & of CYP3A4 & CYP3A4 or inhibitors/ & reported & CYP3A4 or inhibitors/ \\
Routine coagulation & None & None & inducers of P-gP & & inducers of P-gP \\
monitoring & & & None & None
\end{tabular}

Abbreviations: P-gp, P-glycoprotein; CYP3A4, cytochrome P450 3A4.

respectively, vs $0.74 \% ; P<0.05$ for both dabigatran-dose groups compared to warfarin).

Despite similar efficacy and an enhanced safety profile for dabigatran, warfarin demonstrated superior tolerability relative to dabigatran. A total of $15 \%$ and $21 \%$ of patients receiving $110 \mathrm{mg}$ dabigatran discontinued use by the end of the first and second years, respectively. A similar proportion of patients, $16 \%$ and $21 \%$, receiving $150 \mathrm{mg}$ dabigatran discontinued the study medication at 1 and 2 years, respectively, compared to discontinuation rates of $10 \%$ at 1 year and $17 \%$ at 2 years in warfarin-treated patients $(P<0.001)$. This difference was attributed to higher rates of dyspepsia with dabigatran compared to warfarin (11.8\% and $11.3 \%$ for the $110 \mathrm{mg}$ and $150 \mathrm{mg}$ groups, respectively, vs 5.8\% for warfarin; $P<0.001$ for both comparisons).

\section{Direct factor $\mathbf{X a}$ inhibitors Apixaban}

Apixaban is an oral, direct, reversible inhibitor of factor $\mathrm{Xa}$ that after ingestion reaches peak plasma concentrations between 1 and 3 hours (Table 2). ${ }^{15}$ The observed half-life of apixaban is $8-13$ hours. In addition, apixaban is predominantly metabolized via the liver, and approximately $25 \%$ of drug is excreted renally.

Apixaban was evaluated in patients with nonvalvular AF in the phase III AVERROES (Apixaban Versus Acetylsalicylic Acid [ASA] to Prevent Stroke in Atrial Fibrillation Patients Who Have Failed or Are Unsuitable for Vitamin K Antagonist Treatment: A Randomized Double Blind Trial) trial. ${ }^{12}$ In AVERROES, 5,599 patients with nonvalvular $\mathrm{AF}$ as well as at least one risk factor for ischemic stroke, and who were considered "unsuitable" for VKA therapy, were randomized in a prospective, double-blinded fashion to either apixaban $5 \mathrm{mg}$ twice daily or aspirin therapy. The mean follow-up was roughly 1 year. In AVERROES, the rate of stroke or systemic embolism was significantly reduced in apixaban-treated patients compared to aspirintreated patients $(1.65 \%$ vs $3.7 \%$ per year, hazard ratio [HR] $0.45,95 \%$ confidence interval $[\mathrm{CI}] 0.32-0.62 ; P<0.001)$. A nonsignificant reduction in mortality was also observed with apixaban therapy relative to the aspirin control group (3.5\% vs $4.4 \%$ per year, HR 0.79 , 95\% CI $0.62-1.02$; $P=0.07)$. In addition, the risk of first cardiovascular hospitalization was significantly reduced in apixaban-treated patients compared to those receiving aspirin (12.6\% vs $15.9 \%$ per year, $P<0.001)$. Finally, treatment with apixaban was associated with a similar risk of major bleeding (1.4\% vs, $1.2 \%$ per year, HR $1.13,95 \%$ CI $0.74-1.75 ; P=0.57)$ as well as intracranial bleeding $(0.4 \%$ vs $0.4 \%$, HR $0.85,95 \% \mathrm{CI}$ $0.38-1.90 ; P=0.69)$ compared to aspirin.

Apixaban was also investigated and compared directly to standard warfarin therapy in the phase III ARISTOTLE (Apixaban for the Prevention of Stroke in Subjects with Atrial Fibrillation) trial. ${ }^{9}$ In ARISTOTLE, 18,201 patients with $\mathrm{AF}$ and at least one risk factor for thromboembolism were randomized to either apixaban $5 \mathrm{mg}$ twice daily or warfarin (goal INR 2-3). Both prescribers and patients were blinded to treatment. The median follow-up was 1.8 years, after which the rate of the primary efficacy end point (a composite of stroke or systemic embolism) was reduced in apixaban-treated patients compared to those treated with warfarin $(1.27 \%$ vs $1.60 \%$ per year, HR $0.79,95 \%$ CI $0.66-0.95 ; P<0.001$ for noninferiority, $P=0.01$ for superiority). In addition, apixaban demonstrated an enhanced safety profile compared to warfarin, as the rate of major bleeding $(2.13 \%$ vs $3.09 \%$, HR $0.69,95 \%$ CI $0.60-0.80 ; P<0.001)$ as well as the rate of all-cause mortality were significantly reduced with apixaban compared to warfarin $(3.52 \%$ vs $3.94 \%$, HR $0.89 ; 95 \%$ CI $0.80-0.99 ; P<0.047)$. A $58 \%$ relative risk reduction in the risk of intracranial hemorrhage was also observed with apixaban compared to treatment with warfarin $(0.33 \%$ vs $0.80 \%$ per year, HR 0.42 , 95\% CI 0.30-0.58; $P<0.001$ ). 


\section{Rivaroxaban}

Rivaroxaban is another oral, reversible, direct-acting factor $\mathrm{Xa}$ inhibitor that has recently received regulatory approval for the prevention of stroke or systemic embolization in patients with nonvalvular AF. Similar to apixaban, rivaroxaban is rapidly absorbed upon ingestion and reaches peak plasma concentrations in 2-4 hours (Table 2) ${ }^{17}$ The half-life of rivaroxaban is $5-13$ hours. ${ }^{15}$ In addition, rivaroxaban is initially metabolized in the liver and subsequently excreted by both renal and fecal routes. Approximately two-thirds of the ingested dose is metabolized via hepatic CYP450 enzymes. ${ }^{17-19}$ Fifty percent of these metabolites are excreted via the kidneys, with the rest eliminated in the feces. Additionally, one-third of the ingested rivaroxaban dose is excreted renally as unmodified active drug.

The role of rivaroxaban for anticoagulation in patients with non-valvular AF was explored in the phase III ROCKET AF (Efficacy and Safety Study of Rivaroxaban with Warfarin for the Prevention of Stroke and Noncentral Nervous System Systemic Embolism in Patients with Non-valvular Atrial Fibrillation) trial. ${ }^{10}$ In ROCKET AF, 14,264 patients with a CHADS 2 score of 2 or greater were randomized to either rivaroxaban $20 \mathrm{mg}$ daily or warfarin (target INR 2-3). The dose of rivaroxaban was reduced to $15 \mathrm{mg}$ daily in patients with creatinine clearance of $30-49 \mathrm{~mL} /$ minute. Both prescribers and patients were blinded to treatment. The median follow-up was 1.9 years, after which the rate of stroke and non-central nervous system thromboembolism was reduced with rivaroxaban compared to warfarin $(1.7 \%$ vs $2.2 \%$ per year, HR 0.79 , 95\% CI $0.66-0.96 ; P<0.001$ for noninferiority). In the intention-to-treat analysis, treatment with rivaroxaban was noninferior to warfarin therapy for the prevention of stroke (2.1\% vs $2.4 \%$, HR $0.88,95 \%$ CI $0.74-1.03 ; P<0.001$ for noninferiority, $P=0.12$ for superiority). Additionally, the rate of major and clinically relevant nonmajor (CRNM) bleeding was similar between rivaroxaban and warfarin (14.9\% vs $14.5 \%$, HR 1.03 , 95\% CI $0.96-1.11 ; P=0.44$ ). Of note, relative to warfarin-treated patients, rivaroxaban treatment was associated with a $33 \%$ relative reduction in the risk of intracranial hemorrhage (HR $0.67,95 \% \mathrm{CI}$ $0.31-0.79 ; P=0.003)$ and a $50 \%$ relative risk reduction in the risk of fatal bleeding (HR 0.50; 95\% CI 0.31-0.79; $P=0.003)$. In addition, a planned secondary analysis found the treatment effect of warfarin was consistent across dosing groups based on renal function, indicating the dose reduction to $15 \mathrm{mg}$ daily in this patient population was equally safe and effective. ${ }^{20}$

\section{Betrixaban}

Betrixaban is an oral, direct factor Xa inhibitor with a half-life of approximately 19 hours (Table 2). ${ }^{15}$ It is rapidly absorbed, reaching peak plasma concentrations in 3-4 hours after administration. ${ }^{14}$ Oral bioavailability is approximately $34 \%$ and protein binding roughly $60 \%$. Betrixaban is primarily excreted unchanged via the biliary system; however, approximately $17 \%$ is excreted via the kidneys.

Betrixaban was studied in patients with nonvalvular AF in the EXPLORE-Xa (Phase 2 Study of the Safety, Tolerability and Pilot Efficacy of Oral Factor Xa Inhibitor Betrixaban Compared to Warfarin) trial. ${ }^{14}$ EXPLORE-Xa was a doseranging study in which 508 patients were randomized to receive 40,60 , or $80 \mathrm{mg}$ once daily or unblended warfarin with a target INR of 2.0-3.0. The primary outcome was the time to occurrence of major or CRNM bleeding. The secondary outcomes included any bleeding (major, CRNM, and any other reported bleeding), as well as time to occurrence of death, ischemic or nonischemic stroke, myocardial infarction (MI), or other systemic embolism.

Patients were randomized in a 1:1:1:1 allocation to betrixaban 40,60 , or $80 \mathrm{mg}$ orally, once daily, or warfarin. Randomization to betrixaban or warfarin was not blinded, but the betrixaban dose was double-blinded. Subjects were assessed at screening, and at weeks $0,1,2,4,8$, and 12, and then every 2 months thereafter for a minimum of 90 days and a maximum of 1 year.

The rates of the primary outcome were lowest in the betrixaban $40 \mathrm{mg}$ group (HR 0.14 vs warfarin, $P$-value unadjusted for multiple testing $=0.04,95 \%$ CI $0.017-1.135$ ). The betrixaban $60 \mathrm{mg}$ and $80 \mathrm{mg}$ daily groups had rates similar to warfarin. The rates of any bleeding were significantly less for betrixaban $40 \mathrm{mg}$ and $80 \mathrm{mg}$ compared with warfarin $(95 \%$ CI $0.301-0.856$ for $40 \mathrm{mg}$ vs warfarin and $0.332-0.914$ for $80 \mathrm{mg}$ vs warfarin; $P=0.01$ and 0.02 , respectively).

There was one ischemic stroke in the betrixaban $60 \mathrm{mg}$ and $80 \mathrm{mg}$ groups and none in the betrixaban $40 \mathrm{mg}$ or warfarin groups. There were two deaths, both vascular: one in the betrixaban $40 \mathrm{mg}$ group and one in the warfarin group. There were no MIs, systemic embolic events, or pulmonary embolism events during the study. Similar rates of serious adverse events were observed across all groups $(9.4 \%, 9.4 \%$, $8.7 \%$, and $0.4 \%$ for betrixaban $40 \mathrm{mg}, 60 \mathrm{mg}, 80 \mathrm{mg}$, and warfarin groups, respectively). Significantly higher rates of diarrhea were observed for the pooled betrixaban groups compared to the warfarin group ( $6.0 \%$ vs $0.8 \%)$, and rates of premature discontinuation for any cause were higher with 
betrixaban compared to warfarin ( $8.9 \%$ vs $6.3 \%)$. The percentages of patients with elevated alanine aminotransferase greater than three times the upper limit of normal on betrixaban and on warfarin were $1.8 \%$ and $0.8 \%$, respectively. No dose-dependent increase in alanine aminotransferase was observed with betrixaban.

\section{Edoxaban}

Edoxaban is an oral, direct-acting factor Xa inhibitor with a half-life of 6-11 hours (Table 2). ${ }^{21,22}$ It is rapidly absorbed after ingestion, reaching peak plasma concentrations within $1-2$ hours. The oral bioavailability of edoxaban is at least $50 \%$, and approximately $35 \%$ is excreted via the kidneys, with the rest excreted in the feces.

Edoxaban has been evaluated in patients with nonvalvular AF in the phase III EngageAFTIMI48 (Global Study to Assess the Safety and Effectiveness of Edoxaban [DU-176b] vs Standard Practice of Dosing with Warfarin in Patients with Atrial Fibrillation) trial (Table 1). ${ }^{23}$ Results of this trial are pending at this time; however, phase II has previously been published, providing some insight into the efficacy and safety of this agent in this patient population. ${ }^{13}$

In this dose-ranging, 12-week parallel-group, multicenter, prospective, phase II study, 1,146 patients with nonvalvular atrial fibrillation with a $\mathrm{CHADS}_{2}$ score of at least 2 and a creatinine clearance greater than $30 \mathrm{~mL} /$ minute were randomized in a 1:1:1:1:1 ratio to oral edoxaban $30 \mathrm{mg}$ daily, $30 \mathrm{mg}$ twice daily, $60 \mathrm{mg}$ daily, $60 \mathrm{mg}$ twice daily, or warfarin with a target INR of 2.0-3.0. Participants and investigators were blinded to the edoxaban dose, but warfarin was administered open-label. The primary outcomes were the composite of major and/or CRNM bleeding and elevated hepatic enzymes and/or bilirubin. Stroke and systemic embolization were secondary end points. Of note, only 889 patients completed the study.

Major plus CRNM bleeding occurred in 3.2\% of patients randomized to warfarin compared to $3.0 \%(P=1), 7.8 \%$ ( $P=0.029), 3.8 \%(P=0.807)$, and $10.6 \%(P=0.002)$ in the edoxaban $30 \mathrm{mg}$ daily, $30 \mathrm{mg}$ twice-daily, $60 \mathrm{mg}$ daily, and $60 \mathrm{mg}$ twice-daily groups. No significant differences were observed in hepatic enzyme or bilirubin elevations between the edoxaban and warfarin groups. The safety profiles of edoxaban $30 \mathrm{mg}$ and $60 \mathrm{mg}$ daily regimens were similar to warfarin, whereas the twice-daily dose regimens were associated with significantly more bleeding. Therefore, only the $30 \mathrm{mg}$ and $60 \mathrm{mg}$ daily dose regimens will be assessed in Engage AFTIMI $48 .^{23}$

\section{What can we learn from the clinical trials?}

In light of the recent increase in clinical trial data and regulatory approval of novel oral anticoagulants for use in patients with nonvalvular AF, application to clinical practice has become an increasingly germane issue. While betrixaban and edoxaban are currently under development for this clinical application, practical conclusions are difficult to draw for these agents, given their relatively preliminary trial findings. Therefore, this discussion will be limited to those agents that have published phase III data or have received regulatory approval for use in this patient population.

The collective results of the phase III trials with dabigatran, apixaban, and rivaroxaban signify the advent of potentially safer and more efficacious oral anticoagulants. However each of these agents, including warfarin, have distinct pharmacological characteristics that should be considered when contemplating initiating one of these agents. In addition, the clinical trials possess significant variability and nuances that may provide some insights for translation to clinical practice.

While RE-LY, ARISTOTLE, and ROCKET AF provide significant clinical data regarding the use of these novel oral anticoagulants compared to warfarin, the recent regulatory approvals of dabigatran, apixaban, and rivaroxaban present new challenges to clinicians managing patients with AF. In order to make informed decisions when choosing an anticoagulant, clinicians should be aware of several distinct differences in trial design between RE-LY, ARISTOTLE, and ROCKET AF.

While both ARISTOTLE and ROCKET AF were randomized, double-blinded trials, as mentioned previously, RE-LY utilized a PROBE design in which those randomized to warfarin were not blinded to treatment assignment. ${ }^{10-12}$ Although it is difficult to ascertain objectively how this difference may have affected outcomes in the respective trials, clinicians should be aware of several potential sources of bias inherent in the differing trial designs. In open-label trials, central adjudication committees can be utilized to evaluate possible end-point events independent from study sites and blinded to subject treatment allocation. This is a characteristic of a PROBE design, where medications are dispensed in an openlabel fashion while end points are adjudicated by a committee blinded to subject allocation, and helps eliminate subjectivity with regard to outcome assessment. Conceivably, an openlabel design allows management of intercurrent events based on the specific intrinsic characteristics of the anticoagulant, rather than a broad management for all patients as if they 
were on warfarin. This type of management is therefore more likely to represent true differences accurately in the management of warfarin and the comparator anticoagulant in daily practice. ${ }^{24}$

Generally, a blinded trial is assumed to be less prone to bias than an open-label trial, as blinding limits the effects of knowledge of treatment allocation on postrandomization treatment decisions and outcome reporting. ${ }^{25}$ However, blinded trials are not always possible, due to cost considerations and complexity in design. In addition, double-blinded trials may introduce other sources of bias in their own right, such as biases in patient selection by excluding patients for whom complicated double-dummy medication regimens are deemed too burdensome. In addition, patient recruitment can be easier in an open-label trial compared to a doubleblinded trial, as was observed with RE-LY compared to ROCKET AF. The difference in design between open-label and double-blind trials may also lead to differences in patient populations due to subjective preselection protocols. ${ }^{26}$

Although the patient populations in RE-LY and ARISTOTLE were quite similar, ROCKET AF was comprised of a significantly higher-risk patient population. ${ }^{27} \mathrm{In}$ ROCKET AF, $87.0 \%$ of patients had a $\mathrm{CHADS}_{2}$ score of $3-6$, whereas only $32.5 \%$ and $30.2 \%$ of patients in RE-LY and ARISTOTLE, respectively, had CHADS 2 scores of 3-6. Also, $54.8 \%$ of patients in the ROCKET AF cohort had a prior history of stroke, transient ischemic attack, or systemic embolism compared to $20.0 \%$ and $19.4 \%$ in RE-LY and ARISTOTLE, respectively. The prevalence of heart failure, hypertension, and diabetes was also higher in the ROCKET AF cohort compared to the patient populations in RE-LY and ARISTOTLE.

The higher-risk patient population studied in ROCKET AF has several implications that should be considered when interpreting the results of the clinical trials involving novel oral anticoagulants. Perhaps not surprisingly, the rate of all-cause mortality was higher in ROCKET AF compared to ARISTOTLE and RE-LY (4.95\% per year vs 3.94\% and $4.13 \%$, respectively). ${ }^{9-11}$ An increase in the primary efficacy end points was also observed in the control groups in ROCKET AF compared to ARISTOTLE and RE-LY (2.4\% per year vs $1.6 \%$ and $1.69 \%$, respectively).

In addition, although the time in therapeutic range (TTR) observed with warfarin was lower in ROCKET AF compared to that observed in RE-LY or ARISTOTLE (median TTRs $58 \%, 67 \%$, and $66 \%$, respectively), this may be a direct result of the patient populations in the individual trials, as TTR decreases with increasing $\mathrm{CHADS}_{2}$ score. ${ }^{28}$ In addition, the presence of individual $\mathrm{CHADS}_{2}$ components, including heart failure, hypertension, and diabetes, all of which were higher in ROCKET AF compared to RE-LY and ARISTOTLE, are also associated with lower TTR. It should be noted that the effect of rivaroxaban on the primary efficacy end point was consistent across all TTR quartiles, as was the effect on intracranial hemorrhage, although treatment effect was greater in those with lower TTR values. ${ }^{29}$ However a significant interaction was observed between TTR and major or CRNM bleeding, as well as for major bleeding, with rivaroxaban demonstrating a lower HR with the lowest quartile TTR and a higher HR in centers with the highest TTR.

\section{Patient-specific issues}

One major advantage of each of these novel oral anticoagulants is the lack of need for onerous, routine laboratory monitoring and dose titration, as is required for warfarin. In addition, rivaroxaban's once-daily regimen may be preferable over the twice-daily regimens of dabigatran or apixaban for certain patients. However providers should also consider that warfarin's relatively longer half-life may provide more stability of anticoagulation compared to the novel oral anticoagulants in patients for whom medication compliance is an issue due to its longer half-life.

\section{Elderly}

The elderly are a subpopulation of patients who may receive the greatest benefit from these novel oral anticoagulants relative to warfarin. In RE-LY, the effects of dabigatran were similar regardless of patient baseline characteristics, including age. ${ }^{11}$ It should be noted, though, that a subsequent secondary analysis of the data from RE-LY suggested a nominal trend towards more extracranial bleeding in patients age 75 years and older treated with $150 \mathrm{mg}$ dabigatran compared to warfarin therapy. ${ }^{30}$ Despite this observation, age was not observed to exert an effect on the beneficial outcomes associated with dabigatran relative to warfarin for the risk of intracranial bleeding. Additionally, although the $110 \mathrm{mg}$ dose did not receive regulatory approval by the US FDA, this dose is associated with significantly less major bleeding $(4.43 \%$ vs $5.10 \%, P<0.001$ for interaction with age) in elderly patients ( 75 years and older) compared to the $150 \mathrm{mg}$ dose.$^{30}$ This dose was approved by the European Medicines Agency (EMA), and is the recommended dose in patients 75 years and older, according to the EMA. ${ }^{31}$

Similar to dabigatran, the efficacy of apixaban in elderly patients remained consistent compared with warfarin ${ }^{9}$ and aspirin. ${ }^{12}$ In addition, major bleeding was lower with 
apixaban compared to warfarin ${ }^{9}$ and similar to that observed with aspirin therapy. ${ }^{12}$ Similar efficacy results were reported for rivaroxaban in elderly patients in ROCKET AF, although the overall bleeding rate was increased slightly with rivaroxaban compared to warfarin. ${ }^{10,32}$ It should be noted, however, that the risk-benefit ratio for rivaroxaban was consistently favorable across all age-groups.

\section{Renal dysfunction}

Warfarin is primarily excreted through the metabolic enzymatic activity of the liver; however, data exist that suggest dose reductions may be necessary in patients with renal dysfunction. ${ }^{33}$ For instance, results from the Pharmacogenetic Optimization of Anticoagulation Therapy cohort indicated that patients with moderate-to-severe renal dysfunction might have decreased dosing requirements with warfarin compared to patients with normal renal function. ${ }^{34}$ Because patients with end-stage renal disease, including those requiring hemodialysis, were excluded from RE-LY, AVERROES, and ROCKET AF, warfarin remains the drug of choice in this group of patients.

The primary mechanism of excretion of dabigatran is via the renal system. ${ }^{15}$ Therefore, this agent should be used with caution in patients with renal dysfunction. Dabigatran is contraindicated in patients with an estimated glomerular filtration rate (eGFR) less than $15 \mathrm{~mL} /$ minute. $^{8}$ For those with severe renal dysfunction characterized by a creatinine clearance of 15-30 $\mathrm{mL} /$ minute, the dose approved by the US FDA is reduced to $75 \mathrm{mg}$ daily. ${ }^{35}$ This dose was not evaluated in RE-LY, as patients with an estimated eGFR less than $<15 \mathrm{~mL} /$ minute were excluded from the study. ${ }^{11}$ Despite these considerations, no significant interactions were reported for either the primary efficacy or safety end points in RE-LY when stratified by renal function, suggesting this agent does indeed remain a viable alternative to warfarin in patients with moderate-to-severe renal dysfunction. ${ }^{11}$ Of note, the recommended dose according to the EMA in patients with an eGFR between 30 and $50 \mathrm{~mL} /$ minute is $110 \mathrm{mg}$ twice daily. ${ }^{31}$

For apixaban, patients with a creatinine clearance of less than $25 \mathrm{~mL} / \mathrm{minu}$ were excluded from ARISTOTLE. ${ }^{9}$ However, in both ARISTOTLE and AVERROES, the dose was reduced to $2.5 \mathrm{mg}$ twice daily in patients who had evidence of decreased renal clearance. ${ }^{36,37}$ When stratified by renal function, apixaban reduced the rates of stroke, death, and major bleeding compared to warfarin consistently across all levels of renal function. ${ }^{38}$

Because nearly $66 \%$ of an oral rivaroxaban dose is excreted via the renal system $(50 \%$ as unchanged drug and $50 \%$ as inactive metabolites), ${ }^{15}$ special dosing considerations are warranted in patients with decreased creatinine clearance. Patients with an eGFR less than $30 \mathrm{~mL} /$ minute were excluded from ROCKET AF. ${ }^{10}$ Patients with an eGFR between 30 and $49 \mathrm{~mL} /$ minute received a reduced dose of $15 \mathrm{mg}$ daily. Analysis of this patient cohort demonstrated the treatment effect of rivaroxaban was consistent across all dosing groups in ROCKET AF, indicating this reduced dose retains the safety and efficacy profile observed with $20 \mathrm{mg}$ daily in patients with an eGFR between 30 and $40 \mathrm{~mL} /$ minute. $^{20}$

\section{Patients at high risk for bleeding events}

One major consideration with oral anticoagulant therapy is the individual patient's bleeding risk. Several distinct models or scoring systems, such as the HAS-BLED scoring system, were established in order to quantify an individual patient's bleeding risk. ${ }^{39}$ For patients at low or moderate risk of bleeding complications, any of the approved oral anticoagulants may be appropriate for use. However, for the higher-risk patients, the trial data suggest these novel oral anticoagulants may have distinct advantages over warfarin therapy with regard to bleeding outcomes. ${ }^{9-11}$

In RE-LY, treatment with $150 \mathrm{mg}$ dabigatran was associated with a significantly lower risk of life-threatening bleeding complications $(1.45 \%$ vs $1.80 \%, P<0.05),{ }^{11}$ as well as the risk of intracranial hemorrhage compared to warfarin $(0.30 \%$ vs $0.74 \%, P<0.05)$. Subsequent analysis demonstrated a number needed to treat of 250 patients to prevent one bleeding event. ${ }^{40}$ In addition, similar reductions were observed with the $110 \mathrm{mg}$ dose of dabigatran for the risk of life-threatening bleeding and intracranial hemorrhage.

Treatment with apixaban also demonstrated an enhanced safety profile with regard to bleeding outcomes compared to warfarin-treated patients. ${ }^{9}$ Compared to warfarin, apixaban was associated with $58 \%$ and $31 \%$ relative risk reductions in the risk of intracranial hemorrhage and major bleeding, respectively. In addition, apixaban was associated with a 29\% relative risk reduction in the risk of any bleeding compared to warfarin-treated patients.

As noted previously, the patient cohort in ROCKET AF was a significantly higher-risk cohort than that of RE-LY or AVERROES. ${ }^{10}$ In this high-risk patient population, no significant differences were observed in the rates of major and CRNM bleeding between patients receiving rivaroxaban or warfarin. However, rivaroxaban therapy was associated with significant reductions in fatal bleeding $(0.4 \%$ vs $0.8 \%$, HR $0.50,95 \%$ CI $0.31-0.79 ; P=0.003)$ and intracranial hemorrhage $(0.8 \%$ vs $1.2 \%$, HR $0.67,95 \%$ CI $0.47-0.93$; $P=0.02$ ) compared to the warfarin group. 
Currently, the data regarding reversibility of anticoagulant effects of these novel oral anticoagulants are limited; however, the available data suggest that factor Xa inhibitors may be "reversible" with blood-replacement products such as prothrombin complex concentrates. ${ }^{41}$ These same products do not appear to offer the same "reversibility" with dabigatran. The safety and efficacy of activated prothrombin protein complexes, higher doses of prothrombin complex concentrates, and recombinant factor VII in this setting with dabigatran are currently unknown. It has been suggested that activated charcoal may offer the potential for adsorption of recently ingested (within a few hours) doses of dabigatran. ${ }^{42}$ In addition, hemodialysis may be successful in removing approximately two-thirds of the circulating active metabolite of dabigatran within $2-4$ hours. ${ }^{43}$

\section{Triple-antithrombotic therapy}

An increased rate of bleeding complications has been observed in patients with an indication for both dualantiplatelet therapy (DAPT) and an oral anticoagulant. An analysis of one Danish registry of patients discharged following a first hospitalization for AF found an increased HR for combined fatal and nonfatal bleeding in patients receiving DAPT and warfarin (HR 3.70, 95\% CI 2.89-4.76), clopidogrel and warfarin (HR 3.08, 95\% CI 2.32-3.91), aspirin and warfarin (HR 1.83, 95\% CI 1.72-1.96), and aspirin and clopidogrel (HR 1.66, 95\% CI 1.34-2.04) compared to warfarin alone. No elevated risk was observed with aspirin monotherapy (HR $0.93,95 \%$ CI $0.88-0.98$ ) or clopidogrel monotherapy (HR 1.06, 95\% CI 0.87-1.29) compared to warfarin monotherapy. ${ }^{44}$

Several novel oral anticoagulants have been investigated in combination with DAPT. In the RE-DEEM (Dose-Finding Study for Dabigatran Etexilate in Patients with Acute Coronary Syndrome) trial, patients with a recent ST-elevation or non-ST-elevation acute coronary syndrome (ACS) were randomized to DAPT and increasing doses of dabigatran or matching placebo ${ }^{45}$ The addition of dabigatran was associated with a dose-dependent increase in International Society of Thrombosis and Haemostasis-defined major or CRNM bleeding compared to placebo $(3.5 \%, 4.3 \%, 7.9 \%$, and $7.8 \%$ for 50, 75, 110, and $150 \mathrm{mg}$ dabigatran groups, respectively, vs $2.2 \%$ for placebo; $P<0.001$ for trend). In RE-LY, the risk of major bleeding was nearly twice as high in patients receiving aspirin or clopidogrel with dabigatran compared to those receiving dabigatran alone. ${ }^{46} \mathrm{~A}$ consistent increase was also observed in those patients receiving aspirin or clopidogrel in addition to warfarin.
Both apixaban and rivaroxaban have been investigated in randomized, prospective trials in the ACS setting in combination with DAPT. In APPRAISE-2 (Phase III Acute Coronary Syndrome), patients were randomized to DAPT plus apixaban $5 \mathrm{mg}$ twice daily or matching placebo; ${ }^{47} 97 \%$ were taking aspirin, and $81 \%$ were treated with DAPT at the time of randomization. An increase in Thrombolysis in Myocardial Infarction group-defined major bleeding was observed with apixaban therapy compared to placebo (HR 2.59, 95\% CI 1.50-4.46; $P=0.001$ ). The composite of cardiovascular death, MI, or ischemic stroke occurred in $7.5 \%$ of patients receiving apixaban and $7.9 \%$ of placebotreated patients (HR $0.95,95 \%$ CI $0.80-1.11 ; P=0.51$ ). In addition, more fatal $(0.1 \%$ vs $0.0 \%)$ and intracranial $(0.3 \%$ vs $0.1 \%$, HR $4.06,95 \%$ CI $1.15-14.38 ; P=0.03$ ) bleeding events occurred in apixaban-treated patients compared to placebo. Although the clinical scenario investigated in APPRAISE-2 may not be necessarily applicable to patients with a separate indication for DAPT and an oral anticoagulant for AF, further analysis from ARISTOTLE may offer additional information regarding this specific issue.

In ATLAS ACS 2-TIMI 51 (Anti-Xa Therapy to Lower Cardiovascular Events in Addition to Standard Therapy in Subjects with Acute Coronary Syndrome-Thrombolysis In Myocardial Infarction-51) patients were randomized to receive rivaroxaban 2.5 or $5 \mathrm{mg}$ twice daily or matching placebo in combination with DAPT. ${ }^{48}$ Treatment with rivaroxaban resulted in a significant decrease in the rate of the composite outcome of cardiovascular death, MI, or stroke ( $8.9 \%$ vs $10.7 \%$, HR $0.84,95 \%$ CI $0.74-0.96 ; P=0.008$ ) compared to placebo-treated patients. Additionally, the $2.5 \mathrm{mg}$ dose was associated with a reduction in the rate of cardiovascular deaths $(2.7 \%$ vs $4.1 \%$, HR $0.66,95 \%$ CI $0.51-0.86 ; P=0.002)$ and all-cause mortality $(2.9 \%$ vs $4.5 \%$, HR $0.68,95 \%$ CI $0.53-0.87 ; P=0.002$ ) relative to placebo. In contrast to the observed efficacy benefit, an increase in Thrombolysis In Myocardial Infarction group-defined major bleeding not related to coronary artery-bypass grafting $(2.1 \%$ vs $0.6 \%$, HR 3.96, 95\% CI 2.46-6.38; $P<0.001)$ and a dosedependent increase in the risk of fatal bleeding $(0.1 \%$ with $2.5 \mathrm{mg}$ twice daily vs $0.4 \%$ with $5 \mathrm{mg}$ twice daily, $P=0.04$ ) were observed in patients receiving rivaroxaban.

Recently the $2.5 \mathrm{mg}$ dose of rivaroxaban received regulatory approval from the EMA for use in combination with DAPT in the post-ACS setting. ${ }^{49}$ However it should be noted that the dose studied in ATLAS ACS 2 TIMI 51 (and that approved for use by the EMA) was drastically lower than that evaluated in ROCKET AF in the setting of AF. 
Consequently, it is difficult to extrapolate the results of ATLAS ACS 2 TIMI 51 to the broader AF population. In ROCKET AF, treatment with aspirin in addition to rivaroxaban increased the 100-patient-year event rate for major bleeding from 3.02 to 5.82 relative to those receiving rivaroxaban monotherapy. ${ }^{50}$ Among patients receiving DAPT and rivaroxaban or warfarin, the rates of all bleeding subcategories were elevated compared to those receiving only the respective oral anticoagulant. However, no difference in the rate of major bleeding was observed in DAPT-treated patients between the rivaroxaban and warfarin groups.

To date, no data exist detailing concomitant use of any novel oral anticoagulant in combination with newergeneration antiplatelet agents, such as ticagrelor or prasugrel. However, given the data with clopidogrel and novel oral anticoagulants, one might reasonably expect an amplified bleeding risk when these agents are used in combination.

\section{Conclusion}

The results of recent clinical trials, such as RE-LY, ARISTOLE, AVERROES, and ROCKET AF, have identified the clinical potential for agents with either enhanced safety or efficacy relative to VKAs. Agents such as dabigatran, apixaban, and rivaroxaban that possess similar or enhanced efficacy for the prevention of thrombotic complications, as well as possessing attractive pharmacologic properties, represent a significant advancement in the overall pharmacologic management of nonvalvular AF. The presence of multiple agents with unique pharmacological and clinically tested properties affords clinicians the opportunity to tailor therapy to the individual patient. Here, we have highlighted the results of the late-stage clinical trials with these agents, as well as multiple patient-specific instances that may lend favorability to one agent over another. In addition to dabigatran, apixaban, and rivaroxaban, the results of phase III trials are pending for edoxaban. Without comparative clinical trials of these novel agents, prescribers are limited to assessments of the individual clinical trials to make therapeutic choices. A broad understanding of both the pharmacologic properties as well as the details of the specific clinical trials is therefore necessary to make informed treatment decisions regarding these agents.

\section{Disclosure}

The author reports no conflicts of interest in this work.

\section{References}

1. Lloyd-Jones DM, Wang TJ, Leip EP, et al. Lifetime risk for development of atrial fibrillation: the Framingham Heart Study. Circulation. 2004;110(9):1042-1046.
2. Furie KL, Goldstein LB, Albers GW, et al. Oral antithrombotic agents for the prevention of stroke in nonvalvular atrial fibrillation: a science advisory for healthcare professionals from the American Heart Association/American Stroke Association. Stroke. 2012;43(12): 3442-3453.

3. Arboix A, García-Eroles L, Massons JB, Oliveres M, Pujades R, Targa C. Atrial fibrillation and stroke: clinical presentation of cardioembolic versus atherothrombotic infarction. Int $J$ Cardiol. 2000;73(1):33-42.

4. Fuster V, Rydén LE, Cannom DS, et al. 2011 ACCF/AHA/HRS focused updates incorporated into the ACC/AHA/ESC 2006 guidelines for the management of patients with atrial fibrillation: a report of the American College of Cardiology Foundation/American Heart Association Task Force on Practice Guidelines developed in partnership with the European Society of Cardiology and in collaboration with the European Heart Rhythm Association and the Heart Rhythm Society. J Am Coll Cardiol. 2011;57(11):e101-e198.

5. Hart RG, Benavente O, McBride R, Pearce LA. Antithrombotic therapy to prevent stroke in patients with atrial fibrillation: a meta-analysis. Ann Intern Med. 1999;131(7):492-501.

6. Baker WL, Cios DA, Sander SD, Coleman CI. Meta-analysis to assess the quality of warfarin control in atrial fibrillation patients in the United States. J Manag Care Pharm. 2009;15(3):244-252.

7. Ogilvie IM, Newton N, Welner SA, Cowell W, Lip GY. Underuse of oral anticoagulants in atrial fibrillation: a systematic review. Am J Med. 2010;123(7):638-645. e4.

8. Eriksson BI, Quinlan DJ, Eikelboom JW. Novel oral factor Xa and thrombin inhibitors in the management of thromboembolism. Аnnu Rev Med. 2011;62:41-57.

9. Granger CB, Alexander JH, McMurray JJ, et al. Apixaban versus warfarin in patients with atrial fibrillation. $N$ Engl J Med. 2011;365(11): 981-992.

10. Patel MR, Mahaffey KW, Garg J, et al. Rivaroxaban versus warfarin in nonvalvular atrial fibrillation. $N$ Engl J Med. 2011;365(10): 883-891.

11. Connolly SJ, Ezekowitz MD, Yusuf S, et al. Dabigatran versus warfarin in patients with atrial fibrillation. $N$ Engl J Med. 2009;361(12): 1139-1151.

12. Connolly SJ, Eikelboom J, Joyner C, et al. Apixaban in patients with atrial fibrillation. N Engl J Med. 2011;364(9):806-817.

13. Weitz JI, Connolly SJ, Patel I, et al. Randomised, parallel-group, multicentre, multinational phase 2 study comparing edoxaban, an oral factor Xa inhibitor, with warfarin for stroke prevention in patients with atrial fibrillation. Thromb Haemost. 2010;104(3):633-641.

14. Connolly SJ, Eikelboom J, Dorian P, et al. Betrixaban compared with warfarin in patients with atrial fibrillation: results of a phase 2, randomized, dose-ranging study (Explore-Xa). Eur Heart J. 2013; 34(20):1498-1505.

15. Eriksson BI, Quinlan DJ, Weitz JI. Comparative pharmacodynamics and pharmacokinetics of oral direct thrombin and factor xa inhibitors in development. Clin Pharmacokinet. 2009;48(1):1-22.

16. Blech S, Ebner T, Ludwig-Schwellinger E, Stangier J, Roth W. The metabolism and disposition of the oral direct thrombin inhibitor, dabigatran, in humans. Drug Metab Dispos. 2008;36(2):386-399.

17. Davis EM, Packard KA, Knezevich JT, Campbell JA. New and emerging anticoagulant therapy for atrial fibrillation and acute coronary syndrome. Pharmacotherapy. 2011;31(10):975-1016.

18. Weinz C, Radtke M, Schmeer K, Kern A, Pleiss U. In vitro metabolism of BAY 59-7939 - an oral, direct factor Xa inhibitor. Drug Metab Rev. 2004;36 Suppl 1:98.

19. Weinz C, Schwarz T, Pleiss U, et al. Metabolism and distribution of [C-14]BAY 59-7939 - an oral, direct factor Xa inhibitor - in rat, dog and human. Drug Metab Rev. 2004;36 Suppl 1:98.

20. Fox KA, Piccini JP, Wojdyla D, et al. Prevention of stroke and systemic embolism with rivaroxaban compared with warfarin in patients with non-valvular atrial fibrillation and moderate renal impairment. Eur Heart J. 2011;32(19):2387-2394. 
21. Camm AJ, Bounameaux H. Edoxaban: a new oral direct factor xa inhibitor. Drugs. 2011;71(12):1503-1526.

22. Ogata K, Mendell-Harary J, Tachibana M, et al. Clinical safety, tolerability, pharmacokinetics, and pharmacodynamics of the novel factor Xa inhibitor edoxaban in healthy volunteers. J Clin Pharmacol. 2010;50(7):743-753.

23. Ruff CT, Giugliano RP, Antman EM, et al. Evaluation of the novel factor Xa inhibitor edoxaban compared with warfarin in patients with atrial fibrillation: design and rationale for the Effective Anticoagulation with Factor Xa Next Generation in Atrial Fibrillation-Thrombolysis in Myocardial Infarction study 48 (ENGAGE AF-TIMI 48). Am Heart J. 2010;160(4):635-641.

24. Ahrens I, Lip GY, Peter K. What do the RE-LY, AVERROES and ROCKET-AF trials tell us for stroke prevention in atrial fibrillation? Thromb Haemost. 2011;105(4):574-578.

25. Beyer-Westendorf J, Buller H. External and internal validity of open label or double-blind trials in oral anticoagulation: better, worse or just different? J Thromb Haemost. 2011;9(11):2153-2158.

26. Juni P, Altman DG, Egger M. Systematic reviews in health care: assessing the quality of controlled clinical trials. BMJ. 2001;323(7303):42-46.

27. Lip GY, Larsen TB, Skjoth F, Rasmussen LH. Indirect comparisons of new oral anticoagulant drugs for efficacy and safety when used for stroke prevention in atrial fibrillation. J Am Coll Cardiol. 2012;60(8): 738-746.

28. Nelson WW, Choi JC, Vanderpoel J, et al. Impact of co-morbidities and patient characteristics on international normalized ratio control over time in patients with nonvalvular atrial fibrillation. Am J Cardiol. Epub June 22, 2013.

29. US Food and Drug Administration. Rivaroxaban for the prevention of stroke and non-central nervous system (CNS) systemic embolism in patients with atrial fibrillation. 2011. Available from: http://www.fda.gov/ downloads/AdvisoryCommittees/CommitteesMeetingMaterials/Drugs/ CardiovascularandRenalDrugsAdvisoryCommittee/UCM270797.pdf. Accessed July 15, 2013.

30. Eikelboom JW, Wallentin L, Connolly SJ, et al. Risk of bleeding with 2 doses of dabigatran compared with warfarin in older and younger patients with atrial fibrillation: an analysis of the randomized evaluation of long-term anticoagulant therapy (RE-LY) trial. Circulation. 2011;123(21):2363-2372.

31. Camm AJ, Lip GY, De Caterina R, et al. 2012 Focused update of the ESC Guidelines for the management of atrial fibrillation: an update of the 2010 ESC Guidelines for the management of atrial fibrillation. Developed with the special contribution of the European Heart Rhythm Association. Eur Heart J. 2012;33(21):2719-2747.

32. Janssen Pharmaceuticals. Xarelto (rivaroxaban) tablets [prescribing information]. 2011. Available from: http://www.xareltohcp.com/sites/ default/files/pdf/xarelto_0.pdf. Accessed July 15, 2013.

33. Grand'Maison A, Charest AF, Geerts WH. Anticoagulant use in patients with chronic renal impairment. Am J Cardiovasc Drugs. 2005;5(5): 291-305.

34. Limdi NA, Limdi MA, Cavallari L, et al. Warfarin dosing in patients with impaired kidney function. Am J Kidney Dis. 2010;56(5):823-831.

35. Wann LS, Curtis AB, January CT, et al. 2011 ACCF/AHA/HRS focused update on the management of patients with atrial fibrillation (updating the 2006 guideline): a report of the American College of Cardiology Foundation/American Heart Association Task Force on Practice Guidelines. Circulation. 2011;123(1):104-123.

Open Access Journal of Clinical Trials

\section{Publish your work in this journal}

The Open Access Journal of Clinical Trials is an international, peerreviewed, open access journal publishing original research, reports, editorials, reviews and commentaries on all aspects of clinical trial design, management, legal, ethical and regulatory issues, case record form design, data collection, quality assurance and data auditing
36. Eikelboom JW, O'Donnell M, Yusuf S, et al. Rationale and design of AVERROES: apixaban versus acetylsalicylic acid to prevent stroke in atrial fibrillation patients who have failed or are unsuitable for vitamin $\mathrm{K}$ antagonist treatment. Am Heart J. 2010;159(3):348-353. e1.

37. Lopes RD, Alexander JH, Al-Khatib SM, et al. Apixaban for reduction in stroke and other thromboembolic events in atrial fibrillation (ARISTOTLE) trial: design and rationale. Am Heart J. 2010;159(3): 331-339.

38. Hohnloser SH, Hijazi Z, Thomas L, et al. Efficacy of apixaban when compared with warfarin in relation to renal function in patients with atrial fibrillation: insights from the ARISTOTLE trial. Eur Heart $J$. 2012;33(22):2821-2830.

39. Pisters R, Lane DA, Nieuwlaat R, de Vos CB, Crijns HJ, Lip GY. A novel user-friendly score (HAS-BLED) to assess 1-year risk of major bleeding in patients with atrial fibrillation: the Euro Heart Survey. Chest. 2010;138(5):1093-1100.

40. Altman R, Vidal HO. Battle of oral anticoagulants in the field of atrial fibrillation scrutinized from a clinical practice (the real world) perspective. Thromb J. 2011;9:12.

41. Eerenberg ES, Kamphuisen PW, Sijpkens MK, Meijers JC, Buller HR, Levi M. Reversal of rivaroxaban and dabigatran by prothrombin complex concentrate: a randomized, placebo-controlled, crossover study in healthy subjects. Circulation. 2011;124(14):1573-1579.

42. Kaatz S, Kouides PA, Garcia DA, et al. Guidance on the emergent reversal of oral thrombin and factor Xa inhibitors. Am J Hematol. 2012;87 Suppl 1:S141-S145.

43. Stangier J, Rathgen K, Stahle H, Mazur D. Influence of renal impairment on the pharmacokinetics and pharmacodynamics of oral dabigatran etexilate: an open-label, parallel-group, single-centre study. Clin Pharmacokinet. 2010;49(4):259-268.

44. Hansen ML, Sorensen R, Clausen MT, et al. Risk of bleeding with single, dual, or triple therapy with warfarin, aspirin, and clopidogrel in patients with atrial fibrillation. Arch Intern Med. 2010;170(16):1433-1441.

45. Oldgren J, Budaj A, Granger CB, et al. Dabigatran vs placebo in patients with acute coronary syndromes on dual antiplatelet therapy: a randomized, double-blind, phase II trial. Eur Heart J. 2011;32(22): 2781-2789.

46. Boehinger Ingelheim Pharmaceuticals. New drug application (NDA) 22-512: dabigatran etexilate. 2010. Available from: http://www.fda. gov/downloads/advisorycommittees/committeesmeetingmaterials/ drugs/cardiovascularandrenaldrugsadvisorycommittee/ucm 226009 . pdf. Accessed December 27, 2011.

47. Alexander JH, Lopes RD, James S, et al. Apixaban with antiplatelet therapy after acute coronary syndrome. $N$ Engl J Med. 2011;365(8): 699-708.

48. Mega JL, Braunwald E, Wiviott SD, et al. Rivaroxaban in patients with a recent acute coronary syndrome. N Engl J Med. 2012;366(1):9-19.

49. European Medicines Agency. Summary of opinion (post authorisation): Xarelto (rivaroxaban). 2013. Available from: http://www.ema. europa.eu/docs/en_GB/document_library/Summary_of_opinion/ human/000944/WC500140679.pdf. Accessed July 5, 2013.

50. US Food and Drug Administration. FDA draft briefing document for the Cardiovascular and Renal Drugs Advisory Committee (CRDAC): Xarelto (rivaroxaban) tablets. 2011. Available from: http://www.fda. gov/downloads/AdvisoryCommittees/CommitteesMeetingMaterials/ drugs/CardiovascularandRenalDrugsAdvisoryCommittee/ucm270796. pdf. Accessed July 5, 2013.

\section{Dovepress}

methodologies. The manuscript management system is completely online and includes a very quick and fair peer-review system, which is all easy to use. Visit http://www.dovepress.com/testimonials.php to read real quotes from published authors. 